\title{
Untersuchungen über die Clostridium perfringens-Neuraminidase
}

\author{
Erika Balke und Rudolf Drzeniek \\ Institut für Hygiene und Infektionskrankheiten der Tiere und Institut für Virologie \\ der Justus Liebig-Universität Gießen
}

(Z. Naturforschg. 24 b, 599-603 [1969] ; eingegangen am 23. Dezember 1968)

\begin{abstract}
Aus Clostridium perfringens isolierte und gereinigte Neuraminidase wurde näher charakterisiert. Die Sedimentationskonstante beträgt $s_{20}=4,6 \mathrm{~S}$; das Mol.-Gew. wurde durch Gelfiltration zu 56000 bestimmt. Die Neuraminidase läßt sich durch $10^{-3} \mathrm{M}$ KCN oder $0,03 \%$ Rinderserumalbumin stabilisieren. Ihr Temperatur-Optimum liegt bei $50{ }^{\circ} \mathrm{C}$, das $\mathrm{pH}-$ Optimum mit Fetuin als Substrat in $0,1 \mathrm{~m}$ K-Acetatpuffer bei $\mathrm{pH} 5,0$ bis 5,5 .
\end{abstract}

Neuraminidase ( $N$-Acetylneuraminat-glycohydrolase EC 3.2.1.18) konnte bei einer Reihe von Bakterien nachgewiesen werden ${ }^{1-3}$. Am eingehendsten untersucht wurde das Enzym von Vibrio cholerae, das in kristallisierter Form erhalten werden konnte (l. c. $\left.{ }^{4-6}\right)$. Über die Eigenschaften des aus anderen Mikroorganismen gewonnenen Enzyms ist bisher noch wenig bekannt. Die bei Clostridium perfringens gefundene Neuraminidase ${ }^{7}$ konnte von CAssidy ${ }^{8}$ isoliert und partiell gereinigt werden.

In der vorliegenden Arbeit wurde das Enzym aus Clostridium perfringens durch Bestimmung des Mol.Gew. und einiger enzymkinetischer Eigenschaften näher charakterisiert.

\section{Material und Methode}

$N$-Acetylneuraminsäure wurde von der Firma Light u. Co. Limited, Colnbrook, Buckinghamshire, bezogen. Cytochrom c lieferte die Firma Boehringer, Mannheim. Trypsininhibitor aus Sojabohne, Ovalbumin und Rinderserumalbumin (RSA) wurden von der Firma Serva Entwicklungslabor Heidelberg bezogen. Dextranblau erhielten wir von der Firma Pharmacia, Uppsala. Fetuin wurde aus fetalem Kälberserum ${ }^{9}$ dargestellt.

Der Gehalt an glykosidisch gebundener Sialinsäure schwankte in den einzelnen Präparationen von 6,5 bis 6,8 Prozent. Sialolaktose wurde aus Rinderkolostrum nach der von SchNeIR et al. ${ }^{10}$ beschriebenen Methode

1 A. Gottschalk, Biochim. biophysica Acta [Amsterdam] 23, 645 [1957].

2 A. GotTschalk, Glycoproteins. Their Composition, Structure and Function. Elsevier Publication Company, Amsterdam, London, New York 1966.

3 H. Faillard, Aminozucker spaltende Enzyme in: HoppeSeyler Thierfelder, Handbuch der physiologisch- und pathologisch-chemischen Analyse VI b, S. 1234, SpringerVerlag, Berlin, Göttingen, Heidelberg 1966.

${ }^{4}$ G. L. AdA, E. L. French, and P. E. Lind, J. gen. Microbiol. 24, 409 [1961]. dargestellt. Das Druckdialysiergerät M 140, die Lsg60-Membranen und die Kollodiumhülsen lieferte die Membranfiltergesellschaft Göttingen. Clostridium perfringens ATCC 10543 stellte uns dankenswerterweise Dr. G. W. Jourdian zur Verfügung.

Darstellung der Neuraminidase. Die Neuraminidase ( $N$-Acetylneuraminat-glycohydrolase EC 3.2.1.18) aus Clostridium perfringens ATCC 10543 wurde im Prinzip nach der Methode von CAssidy ${ }^{8}$ gewonnen. Die Bakterienkulturen wurden in 2-l-Ansätzen $3-4$ Stdn. bei $37{ }^{\circ} \mathrm{C}$ gezüchtet, danach sofort auf $4{ }^{\circ} \mathrm{C}$ abgekühlt und die Zellen 20 Min. bei $16000 \mathrm{~g}$ abzentrifugiert. Das zwischen 50-85-proz. Ammonsulfatsättigung entstehende Präzipitat wurde gegen bidest Wasser dialysiert, gefriergetrocknet und bei $-30{ }^{\circ} \mathrm{C}$ aufbewahrt. $1,5 \mathrm{~g}$ des gefriergetrockneten Materials (ca. 1,3 g Protein) wurden in $20 \mathrm{ml}$ eines $10^{-3} \mathrm{M}$ Phosphatpuffers, $\mathrm{pH} 6,0$, der $0,5 \% \mathrm{NaCl}$ enthielt, gelöst und bei $100000 \mathrm{~g}$ 1,5 Stdn. zentrifugiert, der Überstand wurde auf die vorbereitete Sephadex-G-75-Säule $(7 \cdot 75 \mathrm{~cm})$ aufgetragen und mit dem gleichen Puffer eluiert. Aufgefangen wurden 40-ml-Fraktionen. Die Hauptmenge des Enzyms erschien im Eluat zwischen 800 und $1150 \mathrm{ml}$. Dem Eluat wurde zur Stabilisierung des Enzyms $10^{-3} \mathrm{M}$ KCN hinzugefügt.

Die Konzentrierung erfolgte durch Druckdialyse mit Hilfe des Druckdialysiergerätes M 140 und der Lsg$60-$ Membran, $15 \mathrm{~cm} \phi$, unter $30 \mathrm{~atm}$ Stickstoff in ca. einer Stunde. Das auf der Membran verbliebene Protein wurde in ca. $30-50 \mathrm{ml}$ einer $10^{-3} \mathrm{M}$ KCN-Lösung aufgenommen und auf $3-5 \mathrm{ml}$ in einer Kollodiumhülse unter Wasserstrahlpumpen-Vakuum eingeengt.

5 E. Mohr u. G. Schramm, Z. Naturforschg. 15 b, 568 [1960].

6 J. Pye and C. C. Curtain, J. gen. Microbiol. 24, 423 [1961].

7 J. F. McCrea, Austral. J. exp. Biol. med. Sci. 25, 127 [1947].

8 J. Cassidy, G. W. Jourdian, and S. Roseman, J. biol. Chemistry 240, 3501 [1965].

9 Colowick, Kaplan, Methods in Enzymology, Academic Press, New York and London 1962, Bd. V, p. 114.

10 M. Schneir, R. J. Winzler u. M. E. Rafelson, JR., Biochem. Prepar. 9, 1 [1962]. 
Hiervon wurde die halbe Menge, ca. $17000 \mu \mathrm{g}$ Protein, auf eine $1,2 \cdot 20 \mathrm{~cm}$ DEAE-Zellulose-Säule aufgegeben und mit einem linearen KCl-Gradienten $(0,025$ bis $0,15 \mathrm{M} \mathrm{KCl}$ ) eluiert. Das Enzym-haltige Eluat, in 2-ml-Fraktionen aufgefangen, wurde wiederum in der Kollodiumhülse eingeengt.

Durch die beschriebene Reinigungsmethode stieg die spezifische Aktivität um den Faktor 470 (von 0,047 Enzymeinheiten $/ \mathrm{mg}$ Protein nach der Ammonsulfatfällung auf 23 Enzymeinheiten/mg Protein im Endprodukt). Die Ausbeute betrug im Durchschnitt 40 Prozent. Das erhaltene Endprodukt besaß keine $N$-Acetylneuraminsäure-Aldolase-Aktivität.

Bestimmung der Neuraminidase-Aktivität. Sie erfolgte durch Inkubation der Clostridium perfringens. Neuraminidase $15 \mathrm{Min}$. bei $37^{\circ} \mathrm{C}$ in $0,1 \mathrm{M} \mathrm{K}$-Acetatpuffer, $\mathrm{pH}$ 6,0. Die Inkubationsmischung enthielt in $0,5 \mathrm{ml}$ außerdem $150 \mu \mathrm{g}$ RSA, 1,5 mg Fetuin, entsprechend $50 \mu \mathrm{g}$ Gesamt-Sialinsäure und $3 \cdot 10^{-4}$ bis $4 \cdot 10^{-3}$ Enzymeinheiten Neuraminidase. Die Bestimmung der freigesetzten Sialinsäure erfolgte nach der von AMINOFF ${ }^{11}$ angegebenen Methode, wobei die Extinktion bei $552 \mathrm{~nm}$ gemessen wurde ${ }^{12}$. Als eine Enzymeinheit wird diejenige Enzymmenge bezeichnet, welche im Testansatz/min soviel Sialinsäure freisetzt, wie der Extinktion von einem $\mu \mathrm{M} N$-Acetylneuraminsäure entspricht. Die Bestimmung der Gesamt-Sialinsäure erfolgte mit der Orcinmethode wie von GotTSCHALK ${ }^{13}$ angegeben.

Proteinbestimmung. Das Protein wurde nach der Methode von LOWRY ${ }^{14}$ bestimmt. Enthielt die Proteinlösung jedoch KCN, so mußte dieses durch Zugabe eines Überschusses von $1 \mathrm{M} \mathrm{HCl}$ im Exsikkator über $\mathrm{KOH}$ entfernt werden.

\section{Ergebnisse}

\section{Enzymkinetische Eigenschaften}

Die Thermostabilität der gereinigten Clostridium perfringens-Neuraminidase (Abb.1) wurde bei $37^{\circ} \mathrm{C}$, in $0,1 \mathrm{~m} \mathrm{~K}$-Acetatpuffer, $\mathrm{pH} 5,5$, ohne Zusatz, sowie mit $300 \mu \mathrm{g} / \mathrm{ml}$ RSA oder $10^{-2}$ bzw. $10^{-3} \mathrm{M} \mathrm{KCN \text {, }}$ gemessen. Es wurde gefunden, daß die Aktivität des ungeschützten Enzyms bei $37{ }^{\circ} \mathrm{C}$ nach 2 Stdn. bereits auf die Hälfte abgesunken war, während die durch Zugabe von Albumin bzw. KCN stabilisierte Neuraminidase noch eine mittlere Aktivität von mindestens $90 \%$ aufwies. Die Stabilität des Enzyms wird auch bei $4{ }^{\circ} \mathrm{C}$ durch die Zugabe von KCN bzw.

11 D. Aminoff, Biochem. J. 81, 384 [1961].

12 R. Drzeniek, J. T. Seto u. R. Rott, Biochim. biophysica Acta [Amsterdam] 128, 547 [1966].

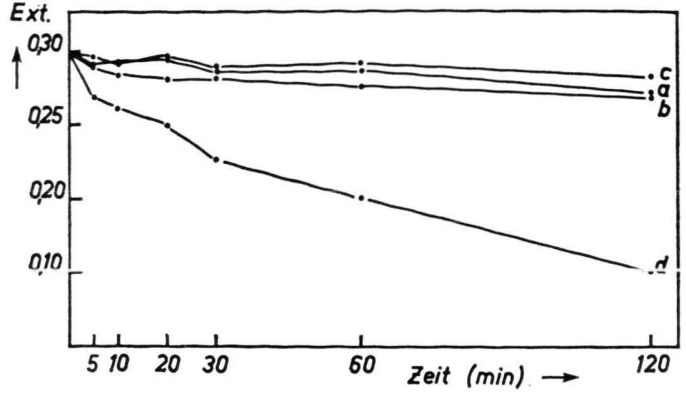

Abb. 1. Thermostabilität der Neuraminidase. 0,092 $\mu \mathrm{g}$ Enzymprotein wurden $5-120 \mathrm{Min}$. bei $37^{\circ} \mathrm{C}$ in 0,1 м Acetatpuffer, $\mathrm{pH} 5,5$, inkubiert und danach die enzymatische Aktivität mit Fetuin als Substrat bestimmt. Enzym geschützt mit : a) $300 \mu \mathrm{g}$ RSA, b) $10^{-2} \mathrm{M} \mathrm{KCN}$ und c) $10^{-3} \mathrm{M} \mathrm{KCN}$, d) ungeschütztes Enzym. Auf der Ordinate sind die Extinktionen der in $0,5 \mathrm{ml}$ freigesetzten Sialinsäure angegeben.

RSA wesentlich erhöht, so daß eine Lagerung im Kühlschrank über 3 Wochen ohne merklichen Aktivitätsverlust möglich ist.

Die Abhängigkeit der Neuraminidase-Aktivität von der Temperatur ist in Abb. 2 angegeben. Nach den durchgeführten Messungen weist die Clostri-

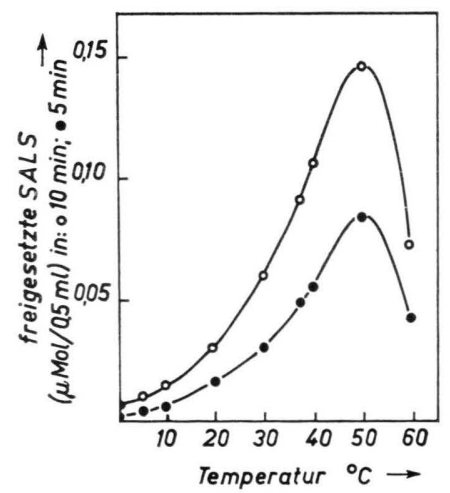

Abb. 2. Temperatur-Optimum der Neuraminidase. 0,39 $\mu \mathrm{g}$ Enzymprotein wurden 5 bzw. 10 Min. mit Fetuin als Substrat in $0,1 \mathrm{M}$ Acetatpuffer $\mathrm{pH} 6,0$ und $150 \mu \mathrm{g} \mathrm{RSA} / 0,5 \mathrm{ml}$ inkubiert und die freigesetzte Sialinsäure (SALS) bestimmt.

dium perfringens-Neuraminidase eine maximale Aktivität bei einer Temperatur von $50{ }^{\circ} \mathrm{C}$ auf. Die Übereinstimmung der bei 5 und 10 Min. gemessenen Werte zeigt, da $\beta$ in diesem Zeitraum bis zu einer Temperatur von $50{ }^{\circ} \mathrm{C}$ keine erfaßbare thermische Denaturierung des Neuraminidase-Moleküls

13 A. Gottschalk, The chemistry and biology of Sialic acids and related substances, Cambridge, University Press, London 1960 , p. 54 .

14 Hoppe-Seyler Thierfelder, Handbuch der physiologischund pathologisch-chemischen Analyse, Springer-Verlag, Berlin, Göttingen, Heidelberg, 1960, Bd. IV, 1, p. 384. 
im Testsystem stattfindet. Das gleiche TemperaturOptimum von $50{ }^{\circ} \mathrm{C}$ fanden MoHr und SCHRAmm ${ }^{5}$ für das Vibrio cholerae-Enzym in $0,05 \mathrm{~m}$ Acetatpuffer, $\mathrm{pH}$ 5,5.

Untersuchungen mit Fetuin und Rinder-Sialolaktose als Enzymsubstrat wurden in $0,1 \mathrm{M}$ Acetatpuffer, $\mathrm{pH}$ 6,0, ausgeführt. Es wurde hierbei für die beiden Substrate von der gleichen Menge glykosidisch gebundener Sialinsäure, $45-50 \mu \mathrm{g}$ pro Versuch, entsprechend $830 \mu \mathrm{g}$ Fetuin bzw. $100 \mu \mathrm{g}$ Rinder-Sialolaktose, ausgegangen. Abb. 3 zeigt, daß die

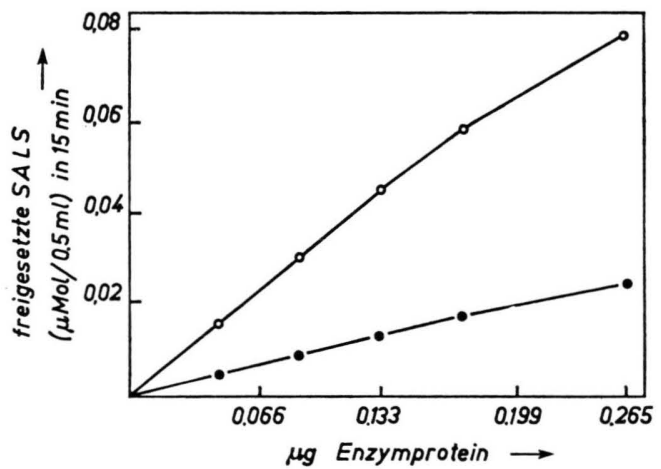

Abb. 3. Enzymaktivität mit Fetuin (o) und Rinder-Sialolaktose (•) als Substrat. In 0,1 M Acetatpuffer, $\mathrm{pH}$ 6,0, waren pro Testansatz $45-50 \mu \mathrm{g}$ glykosidisch gebundene Sialinsäure (SALS), entsprechend $830 \mu \mathrm{g}$ Fetuin bzw. $100 \mu \mathrm{g}$ Sialolaktose vorhanden.

Clostridium perfringens-Neuraminidase aus Fetuin als Substrat dreimal schneller Sialinsäure abspaltet als aus Rinder-Sialolaktose. Entsprechende Untersuchungen mit Neuraminidase aus Vibrio cholerae und Influenzavirus ergaben, daß sich das Enzym von Vibrio cholerae wie das von Clostridium perfringens verhält, das von Influenzavirus dagegen das Fetuin langsamer spaltet als die Rinder-Sialolaktose ${ }^{12}$.

Untersuchungen über das pH-Optimum bei Verwendung von Fetuin als Substrat ergaben, daß die größte Aktivität im Bereich zwischen $\mathrm{pH} 4,0$ bis 6,0 , mit einem schwach ausgebildeten Maximum zwischen pH 5,0 und 5,5 zu finden ist (Abb. 4). Es wurde hierbei unsere Beobachtung berücksichtigt, daß der molare Extinktionskoeffizient der freien Sialinsäure vom $\mathrm{pH}$-Wert des Acetatpuffers abhängig ist. Mit Rinder-Sialolaktose als Substrat fanden CAssidy et al. ${ }^{8}$ in $0,1 \mathrm{M}$ Acetatpuffer die optimale Wirkung der Clostridium perfringens-Neuraminidase bei $\mathrm{pH}$

15 T. Svedberg and K. O. Pedersen, The Ultrazentrifuge, At the Clarendon Press, Oxford 1940.

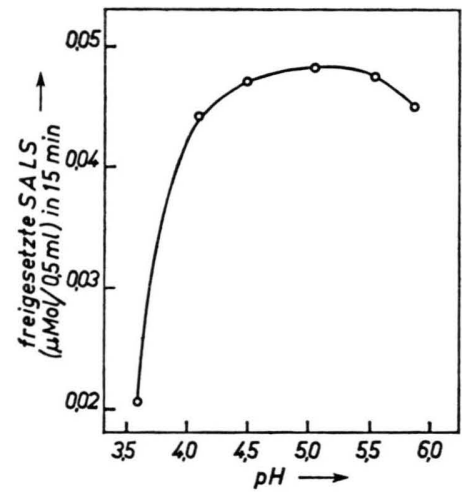

Abb. 4. pH-Optimum der Neuraminidase. 0,128 $\mu \mathrm{g}$ Enzymprotein wurden in $0,05 \mathrm{M} \mathrm{K}$-Acetatpuffer verschiedener $\mathrm{pH}$ Werte mit Fetuin als Substrat 15 Min. bei $37^{\circ} \mathrm{C}$ inkubiert und die freigesetzte Sialinsäure (SALS) gemessen.

4,5. Bekanntlich hängt das pH-Optimum der Neuraminidasen von der Art des verwendeten Substrates $\mathrm{ab}^{5}$.

\section{Sedimentationskonstante und Molekulargewicht}

Die Sedimentationskonstante der Neuraminidase aus Clostridium perfringens wurde mit der von Svedberg und Pedersen ${ }^{15}$ entwickelten Transportmethode (biologische Zentrifugation) wie bereits beschrieben ${ }^{16}$ bestimmt. Sie beträgt in $0,1 \mathrm{~m}$ Acetatpuffer $\mathrm{pH} 6,0 s_{20}=4,6 \pm 0,1 \mathrm{~S}$. Hierbei hatte eine Zugabe von KCN $\left(10^{-3} \mathrm{M}\right)$ oder Cytochrom c (100 $\mu \mathrm{g} / \mathrm{ml}$ ) keinen Einfluß auf die Größe der Sedimentationskonstante.

Das Mol.-Gew. wurde mit Hilfe der Gelfiltrationsmethode ${ }^{17}$ bestimmt. Hierzu wurde eine G-100-Sephadex-Säule, $1,6 \cdot 123 \mathrm{~cm}$, mit $10^{-3} \mathrm{M}$ Phosphatpuffer, dem $0,5 \% \mathrm{NaCl}$ hinzugefügt worden war, benutzt. Die Flußrate der Säule betrug ca. $7 \mathrm{ml} / \mathrm{cm}^{2} / \mathrm{h}$. Die Eluate wurden in Fraktionen von $2 \mathrm{ml}$ aufgefangen. Als Eichsubstanzen zur Ermittlung der Eichgeraden dienten: Cytochrom c, Trypsininhibitor aus Sojabohne, Ovalbumin und Rinderserumalbumin. Sie wurden in einer Menge von je $8 \mathrm{mg} / \mathrm{ml}$ auf die Säule gegeben. Das Ausschlußvolumen der Säule Vo bestimmten wir mehrmals mit je $1 \mathrm{ml}$ einer 0,2-proz. Dextranblaulösung vom $M G=2 \cdot 10^{6}$ durch Messung der Extinktion bei $620 \mathrm{~nm}$. Das Elutionsvolumen Ve von Cytochrom c wurde spektralphotometrisch bei $550 \mathrm{~nm}$, das der anderen Proteine nach LOWRY ${ }^{14}$ bestimmt, die Neuraminidaseaktivität ( $240 \mu \mathrm{g}$ Enzymprotein in $0,5 \mathrm{ml}$ ) wurde wie beschrieben ge-

16 R. Drzeniek, Habilitationsschrift, Gießen 1968.

17 P. ANDREws, Biochem. J. 91, 222 [1964]. 
messen. Ein typisches Elutionsdiagramm der Neuraminidase, das im Prinzip auch für alle anderen verwendeten Substanzen gilt, ist in Abb. 5 darge-

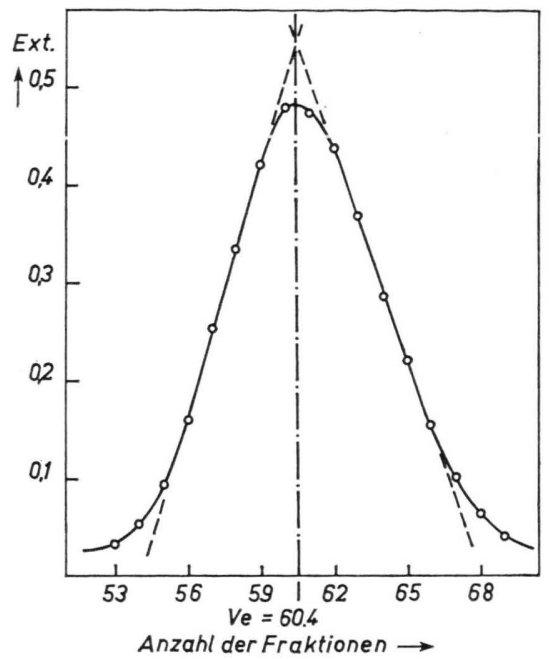

Abb. 5. Diagramm der Neuraminidase an Sephadex-G-100 zur Ermittlung des Elutionsvolumens. Für die Bestimmung des Elutionsvolumens Ve wurden $240 \mu \mathrm{g}$ Enzymprotein in $0,5 \mathrm{ml}$ eines $10^{-3} \mathrm{M}$ Phosphatpuffers, der $0,5 \% \mathrm{NaCl}$ enthielt, auf eine $1,6 \cdot 123 \mathrm{~cm}$ Säule aufgetragen und mit dem gleichen Puffer eluiert. In den aufgefangenen 2-ml-Fraktionen erfolgte die Bestimmung der Neuraminidase-Aktivität mit dem Thiobarbitursäuretest. Auf der Ordinate wurde die Extinktion der freigesetzten Sialinsäure, auf der Abszisse die Anzahl der 2-ml-Fraktionen aufgetragen.

stellt, woraus die Ermittlung des Elutionsgipfels und damit Ve hervorgeht. Aus den experimentell bestimmten Ordinaten der Eichsubstanzen wurde die Eichgerade als Regressionsgerade errechnet und eingezeichnet (Abb.6). Für die Clostridium perfrin-

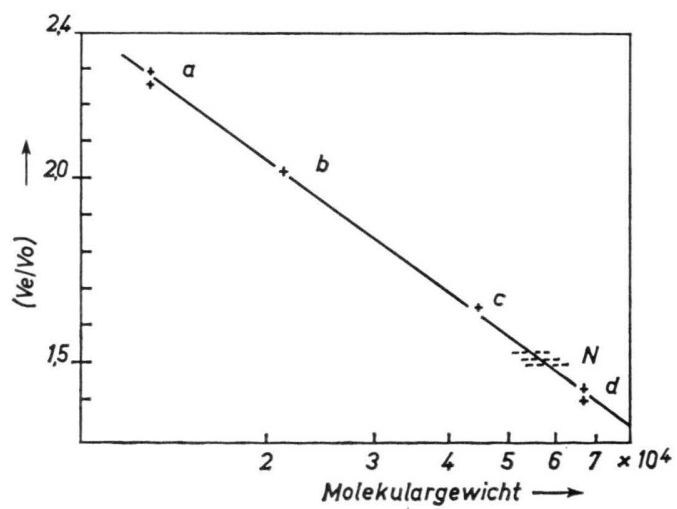

Abb. 6. Mol.-Gew.-Bestimmung an Sephadex-G-100. Eichgerade mit folgenden Eichsubstanzen ermittelt: $\mathrm{a}=$ Cytochrom c $M G=13000, \mathrm{~b}=$ Trypsininhibitor aus Sojabohne $\mathrm{MG}=21500, \mathrm{c}=$ Ovalbumin $M G=45000$ und $\mathrm{d}=$ Rin. derserumalbumin $M G=67000 . \mathrm{N}=$ Schnittpunkte der $(\mathrm{Ve} / \mathrm{Vo})$-Werte der Neuraminidase mit der Eichgeraden.
gens-Neuraminidase erhielten wir die ( $\mathrm{Ve} / \mathrm{Vo})$-Werte $1,53,1,51$ und 1,49 und daraus das mittlere Mol.Gew. von 56000 mit der maximalen Streuung von \pm 6000 .

Die Mol.-Gew.-Bestimmung wurde danach unter völlig veränderten Bedingungen wiederholt. An Stelle des Sephadex-C-100 wurde C-150 verwendet und für die Elution ein 0,01 м Tris-Maleat-Puffer, $\mathrm{pH}$ 6,0 , der $1,5 \% \mathrm{NaCl}$ enthielt, benutzt. Außerdem wurde eine, ebenfalls nach DEAE-Reinigung gewonnene, aber mit $300 \mu \mathrm{g} / \mathrm{ml}$ RSA stabilisierte, über 3 Monate bei $-30^{\circ} \mathrm{C}$ gelagerte NeuraminidasePräparation verwendet, die eine durch die Lagerung bedingte zweifache Aktivitätssteigerung aufwies. Ferner wurde die Neuraminidase-haltige lyophilisierte Ammonsulfatfraktion (35 mg Protein $/ \mathrm{ml}$ ) untersucht. Daneben wurde Bio-Gel P-150 mit 0,05 M Tris-HCl Puffer, $\mathrm{pH} 7,5$, plus $0,1 \mathrm{M} \mathrm{KCl}$ zur Mol.Gew. Bestimmung verwendet. Die in diesen Versuchen ermittelten Mol.-Gew. stimmen mit den an der G-100-Säule erhaltenen Ergebnissen innerhalb der genannten Fehlerbreite überein. Weiterhin zeigen diese Ergebnisse, daß das Enzym mit dem als Stabilisator verwendeten RSA keinen Komplex bildet und durch das Lagern trotz der Aktivitätszunahme keine Veränderung des Mol.-Gew. erfolgt.

\section{Diskussion}

Das Mol.-Gew. der Neuraminidasen ist bis jetzt nur für das Vibrio cholerae-Enzym bestimmt worden. Hierbei wurden zwei verschiedene Werte angegeben. Pye und Curtain ${ }^{6}$ sowie Laver et al. ${ }^{18}$ ermittelten mit Hilfe der Ultrazentrifuge bzw. Endgruppenanalyse ein Mol.-Gew. von 90000 und eine Sedimentationskonstante $s_{20}=5,4 \mathrm{~S}$. Im Gegensatz hierzu bestimmten MoHr und Schramm ${ }^{5}$ die Sedimentationskonstante bei $\mathrm{pH} 8,5$ zu $s_{20}=1,3 \mathrm{~S}$ und extrapolierten auf ein Mol.-Gew. von 8000. Mit der Transportmethode wurde eine Sedimentationskonstante von 5,4 S für Vibrio cholerae-Neuraminidase (l. c. ${ }^{19}$ ) erhalten, was mit dem auf anderem Wege (s. o.) erhaltenen Wert übereinstimmt.

Unsere Untersuchungen ergaben für die Clostridium perfringens-Neuraminidase eine Sedimentationskonstante $s_{20}=4,6 \mathrm{~S}$ bei $\mathrm{pH} 6,0$. Eine Bestim-

18 W. G. Layer, J. Pye, and G. L. Ada, Biochim. biophysica Acta [Amsterdam] 81, 177 [1964].

19 J. T. Seto, R. Drzeniek, and R. Rott, Biochim. biophysica Acta [Amsterdam] 113, 402 [1966]. 
mung des Mol.-Gew. dieses Enzyms mit Hilfe der Gelfiltration ergab einen Wert von 56000.

Im Gegensatz dazu besitzen die Neuraminidasen der Influenzaviren, nach Loslösung vom Virus, eine wesentlich höhere Sedimentationskonstante, und zwar $s_{20}=9-10 \mathrm{~S}^{12}$. Aus elektronenmikroskopischen Untersuchungen geht hervor, daß diese Neuraminidasen ringförmige Strukturen mit einem Durchmesser von $80-90 \AA$ aufweisen und aus Untereinheiten zusammengesetzt sind ${ }^{20}$.

20 R. Drzeniek, H. Frank, and R. Rott, Virology 36, 703 [1968].
Sowohl in der Größe als auch in ihren enzymkinetischen Eigenschaften ähnelt die Clostridium perfringens-Neuraminidase mehr der Vibrio choleraeNeuraminidase als den Virus-Neuraminidasen. Weitere Untersuchungen sind jedoch notwendig um zu zeigen, inwieweit die physikalisch-chemischen und enzymkinetischen Eigenschaften auch anderer Bakterien-Neuraminidasen einander entsprechen.

Frau Herta Perkitny und Fräulein Michaela ORLICH danken wir für ihre ausgezeichnete Mitarbeit. Ein Teil der Untersuchungen wurde durch die Deutsche Forschungsgemeinschaft gefördert.

\title{
Reaktionen der Hydrogenase aus Rhodopseudomonas capsulata im partikelgebundenen und gelösten Zustand ${ }^{1}$
}

\author{
J.-H. Klemme \\ Institut für Mikrobiologie der Universität, Göttingen \\ (Z. Naturforschg. 24 b, 603-612 [1969] ; eingegangen am 11. November 1968)
}

\begin{abstract}
In cell free extracts of Rps. capsulata obtained by exposure of cells to ultrasonic oscillation, about $90 \%$ of the hydrogenase is associated with the particulate chromatophore fraction. The particulate enzyme reacts with methylene blue (MB), menadione, phenazonium methosulfate (PMS), dichlorophenolindophenol (DCPIP), cytochrome c, $p$-benzoquinone (BQ), ferricyanide and $\mathrm{O}_{2}$, but does not react with benzylviologen (BV), pyridinnucleotides and flavinnucleotides. Treatment of chromatophores with sodiumlaurylsulfate inactivates the hydrogenase reaction with PMS, DCPIP, BQ and ferricyanide. The MB-linked or menadione-linked hydrogenase is not destroyed by the detergent. The hydrogenase reaction with BV is increased more than 20 -fold after incubation of the chromatophores with the lipid-dispersing agent. Treatment of chromatophores with acetone and petroleum ether almost completely inactivates the hydrogenase reaction with PMS and BQ. The reaction rate of the DCPIP-linked and the ferricyanide-linked hydrogenase is somewhat decreased, whereas the MB-linked, the menadione-linked and the BV-linked hydrogenase reactions still exhibit about $100 \%$ of the original activity. By extraction of the acetone-treated chromatophores with glycine-NaOH-buffer $(\mathrm{pH} \mathrm{9)}$, about $10-15 \%$ of the particulate hydrogenase is solubilized. The enzyme was 9-fold purified by ammonium sulfate precipitation and chromatography on DEAE-cellulose. The purified enzyme contains no cytochrome. The relative reaction rates of the solubilized enzyme with different electron acceptors are similar to the corresponding reaction rates of the acetonetreated chromatophores. Extraction of chromatophores with n-butanol results in the solubilization of $5-10 \%$ of the particulate enzyme. By extraction of acetone-treated chromatophores with $0,5 \%$ Triton X-100, $40 \%$ of the particulate hydrogenase is solubilized. The fractionation of the extract with ammonium sulfate results in the isolation of a cytochrome c-containing particle which exhibits a 3 -fold increased hydrogenase activity.
\end{abstract}

Die Wasserstoff aktivierenden Enzyme der Bakterien können als im Cytoplasma gelöste oder als an die Cytoplasma-Membran gebundene Proteine vorliegen $^{2-5}$. Als Cofaktoren der löslichen Hydro-

1 Abkürzungen: $\mathrm{BChl}=$ Bacteriochlorophyll, $\mathrm{BV}=\mathrm{Ben}$ zylviologen, $\mathrm{BQ}=p$-Benzochinon, DCPIP $=$ Dichlorphenolindophenol, EDTA = Äthylendiamintetraessigsäure, $\mathrm{FAD}=$ Flavin-Adenin-Dinucleotid, FMN = Flavin-Mononucleotid, $\mathrm{MB}=$ Methylenblau, NAD $(\mathrm{P})=$ NicotinamidAdenin-Dinucleotid (phosphat), PMS = Phenazoniummethosulfat, $R .=$ Rhodospirillum, RSA $=$ Rinderserumalbumin, Rps. = Rhodopseudomonas.

2 B. A. C. Ackrell, R. N. Asato u. H. F. Mower, J. Bacteriol. 92, 828 [1966]. genasen aus Clostridium- und HydrogenomonasArten sind Ferredoxine ${ }^{6}$ bzw. Pyridinnucleotide ${ }^{7}$ genannt worden. Über die mit den partikelgebundenen Hydrogenasen assoziierten Elektronenaccepto-

3 D. H. Bone, Biochem. biophysic. Res. Commun. 3, 211 [1960].

4 U. Eberhardt, Arch. Mikrobiol. 53, 288 [1966].

5 A. Temperli, R. M. Pengra u. P. W. Wilson, Biochim. biophysica Acta [Amsterdam] 38, 557 [1960].

6 W. W. Fredricks u. E. R. Stadtman, J. biol. Chemistry 240, 4065 [1965].

7 L. Packer u. W. Vishniac, Biochim. biophysica Acta [Amsterdam] 17, 153 [1955]. 